\title{
RPA in nuclei and metal clusters
}

\author{
P.-G. Reinhard ${ }^{1}$, S. Weisgerber ${ }^{1}$, O. Genzken ${ }^{2}$, M. Brack ${ }^{3}$ \\ 1 Universität Erlangen, Institut für Theoretische Physik, Staudtstrasse 7, D-91058 Erlangen, Germany \\ 2 C.E.N.G., F-38041 Grenoble, France \\ 3 Universität Regensburg, D-93053 Regensburg, Germany
}

Received: 27 October 1993

\begin{abstract}
We discuss and compare the gross features of resonance excitations in nuclei and metal clusters. We point out the phenomenon of "jellium scaling" which means that different materials for metal clusters all give similar resonance spectra and we discuss the various effects which determine the exact position of the resonance.
\end{abstract}

PACS: $21.60 . J z ; 36.40 .+\mathrm{d}$

The excitation spectra of many-Fermion systems usually display strong resonances, e.g. the giant resonances in nuclei, the plasmons in metal clusters, or zero-sound in liquid ${ }^{3} \mathrm{He}$. These resonances are key signatures for understanding the dynamics of a systems. They are to a good approximation harmonic vibrations and thus can be described very well in the Random-Phase-Approximation (RPA), the microscopic theory of small-amplitude vibrations of a many-Fermion system. It is the aim of this contribution to discuss and compare the gross features of the RPA excitation spectrum of nuclei and metal clusters.

The RPA belongs to the class of mean-field models which becomes obvious by the fact that it can be derived as the small-amplitude limit of time-dependent Hartree-Fock. It represents a vibration on top of a Hartree-Fock ground state. Almost all many-Fermion systems have strong correlations which invalidate a mean-field treatment as ab initio approach. The mean-field treatment requires effective Hamiltonians, a procedure which is known in atomic physics as the Kohn-Sham scheme. We use for the electrons of metal clusters the well known functional of Gunnarson and Lundquist [1]. The ions are treated in the jellium approximation yielding a homogenous positive background which is distributed over a sphere of radius $r_{s} N^{1 / 3}$. For nuclei we employ the Skyrme energy functional, here in particular the parametrization "Skyrme $\mathrm{M}^{*}$ " [2].

There is a wide variety of schemes to derive and compute RPA. We prefer a formulation in terms of an algebra of $1 p h$ operators, coordinate-like $\hat{Q}_{\alpha}$ and momentum-like $\hat{P}_{\alpha}$, which can be optimized in any given subspace or operators. This allows to embedd all conceivable approximations, as e.g. sum rule approach or fluid dynamics, into one and the same scheme by an appropriate choice of the basis operators. For example, the choice of simply one basis state $\hat{Q}_{\alpha}=r^{L} Y_{L M}$ yields the simple sum rule approach, free variation of the radial dependence in $\hat{Q}_{\alpha}=f(r) Y_{L M}$ yields the so called local RPA [3] which is equivalent to an irrotational fluid dynamics, and a choice amongst all possible $1 p h$ excitations $a^{+} a$ restores the full RPA. Besides this flexible switching between various levels of approximation, the scheme provides a very efficient treatment of full RPA in large configuration spaces by a mixing of detailed $1 p h$ states in the region of interest with local excitations over a wide range of excitation energies. For details of the basic formulation see [4], an application to nuclei is discussed in [5], droplets of liquid ${ }^{3} \mathrm{He}$ are considered in [6], and metal clusters will be presented in [7]. We summarize here quickly the results from a comparision of different approaches: the sum rule approximation yields generally an excitation energy which is to high because it forces all strength into one peak, the local RPA provides an appropriate distribution of the overall strength distribution, and fluid dynamics with vorticity gives no improvement compared to local RPA (三 irrotational fluid dynamcis) and produces sometimes even rather misleading details. Altogether, the local RPA is the method of choice; it is inexpensive and it gives the right global pattern of the distribution of strength. For example, the local RPA displays clearly the distinction between a surface Plasmon mode (the so called Mie plasmon) and a volume plasmon mode. It does not reproduce, of course, the detailed splitting over the nearby $1 p h$ spectrum. But at least the fragmentation width can be estimated consistently within the scheme delivering strength functions which are in the average very close to those of full RPA.

In order to get an overview, one needs a compressed way to visualize the spectral properties. We do this by drawing the most important spectral information together in one plot, the energies of the resonance modes in local RPA, the unperturbed $1 p h$ energies, and the particle continuum threshold. A comparison of such spectral properties for nuclei and neutral Na clusters is shown in Fig. 1. The thick vertical line in case of nuclei is to indicate that we have only access to a limited sample of nuclei whereas metal clusters allow, in principle, to study any size up the bulk limit. This 


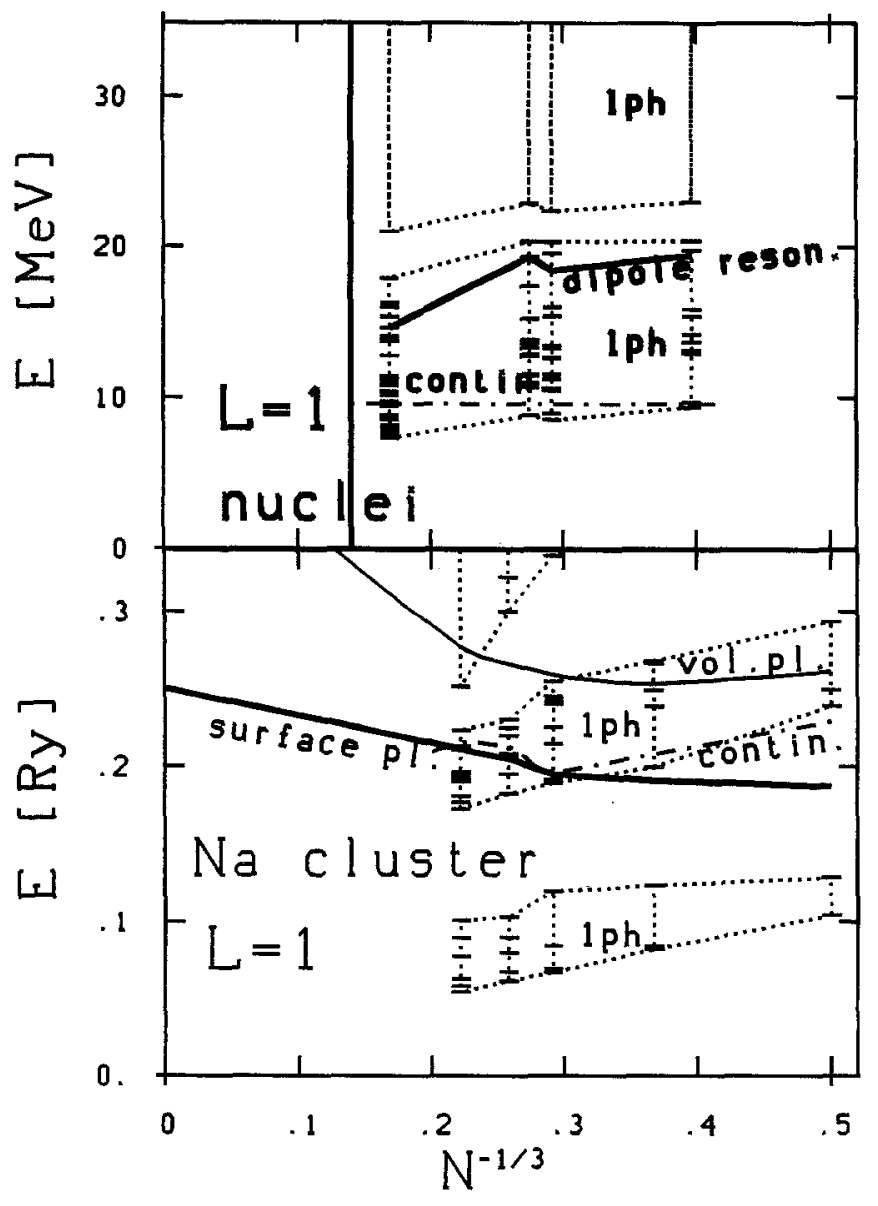

Fig. 1. Spectral properties for $L=1$ modes in nuclei (upper part) and neutral Na clusters (lower part) versus $N^{-1 / 3}$. The thick line is the dominant resonance, the full line show the second resonance (the volume plasmon), the horizontal bars indicate the $1 p h$ energies, and the dotted lines embrace the various bands of $1 p h$ energies. The lowest band consists out of transitions over one shell and the next over 3 shells. The particle continuum threshold (neutrons in case of nuclei, electrons for $\mathrm{Na}$ clusters) is shown by a dash-dotted line

is particularly important for semiclassical expansion which improve with increasing particle number and are usually not converged for the particle numbers provided by nuclei. Both cases display a strong resonance, the dipole giant resonance in nuclei and the surface plasmon in Na clusters. This resonance has a similar structure of collective flow, beeing close to the picture of two charged spheres which oscillate against each other (the so called Goldhaber-Teller mode). But the trends in the resonance energies differ, deacreasing with $N$ for nuclei and slightly increasing for Na clusters. This is due to the range of the force. The short range forces in nuclei deliver collective modes as zero sound with an excitation energy

$\hbar \omega \propto q$

where $q$ is the transferred momentum. The dominant momentum in nuclei is $q \propto 1 / R$ and $R \propto N^{1 / 3}$ yielding the observed decrease approximately as $\hbar \omega \propto N^{-1 / 3}$. The Coulomb force in Na clusters, on the other hand, is of long range delivering the collective excitations as surface plasmon modes with $\hbar \omega=\hbar \omega_{s u r f}+c q^{2}$

where $\omega_{\text {surf }}=\sqrt{e^{2} \rho_{e l} / 3 m}$ is the Mie frequency of the surface plasmon and $c$ is some small constant. Thus the plasmon energy should be essentially constant over the systems which is approximately the case. The bend down observed in Fig. 1 is due to surface effects not contained in the estimate (2). The $1 p h$ bands look similar in both cases. They decrease with $N$ as they should. But the bands are noticeably broader for nuclei. This is due to the large spin-orbit splitting in nuclei. The behaviour of the resonances relative to the bands is much different. The nuclear dipole resonance shares the trend of the $1 p h$ bands and stays always within the bands near the upper bound. This explains that we observe a fragmented resonance in every nucleus, a visible $1 p h$ splitting in the light nuclei and a broad Landau damping in heavy nuclei. The resonance in $\mathrm{Na}$ clusters moves against the direction of the $1 p h$ bands. This gives rise to strong changes of the resonance pattern with the particle number. There is a clean collective peak in $\mathrm{Na}_{8}$ where the resonance falls nicely into a band gap, there is a transitional region between $\mathrm{Na}_{20}$ and $\mathrm{Na}_{40}$ where we see indeed isolated splittings due to interference with one or few $1 p h$ styates at the lower bound of the $1 p h$ band, and there is a broad Landau damped peak in heavier clusters. The width of Landau damping will increase with increasing level density. But it should shrink to zero eventually in the limit $N \rightarrow \infty$ if the $1 p h$ states close to the resonance decouple due to momentum conservation. Thus there should be a transitional region of maximal Landau damping which yet needs to be explored. A final look at the continuum thresholds in Fig. 1 shows that the nuclear resonances are always far above the threshold and thus experience an additional broadening due to neutron escape. The situation is a bit more stable in neutral $\mathrm{Na}$ clusters where the resonance is just below the electron threshold.

There is a most crucial difference which cannot be visualized in one figure: there is only one sort of nuclei but there as a wide choice of materials, characterized by its WignerSeitz radius $r_{s}$, and charge states for metal clusters. At first glance, one would like to ask now for spectral plots as in Fig. 1 for a variety of materials and charges. But it turns out that this is not necessary. We find a property which one could call superficially jellium scaling: within the jellium approximation, all plots of spectral properties versus particle number have essentially the same structure for all reasonable $r_{s}$ and charge states if the energies are properly rescaled. There is always a clean resonance for light clusters, a transitional region at $N=20-40$ and a strong Landau damping for heavy systems. The only exception is the electron continuum threshold which is lifted dramatically for ionized clusters. The consequence is that one should prefer to study positively charged clusters because the essential features of the resonance dynamics are similar as in neutral systems but everything is better bound there and thus more stable. We try to visualize the jellium scaling by a plot of spectral properties versus the Wigner-Seitz radius $r_{s}$. This is done in Fig. 2 for a system in the critical transitional region with $N=20$ electrons. This shows that the spectral relations remain essentially the same throughout all $r_{s}$, except for some tiny differences. This "jellium scaling" holds for the gross properties of the spectra in the resonance region. 


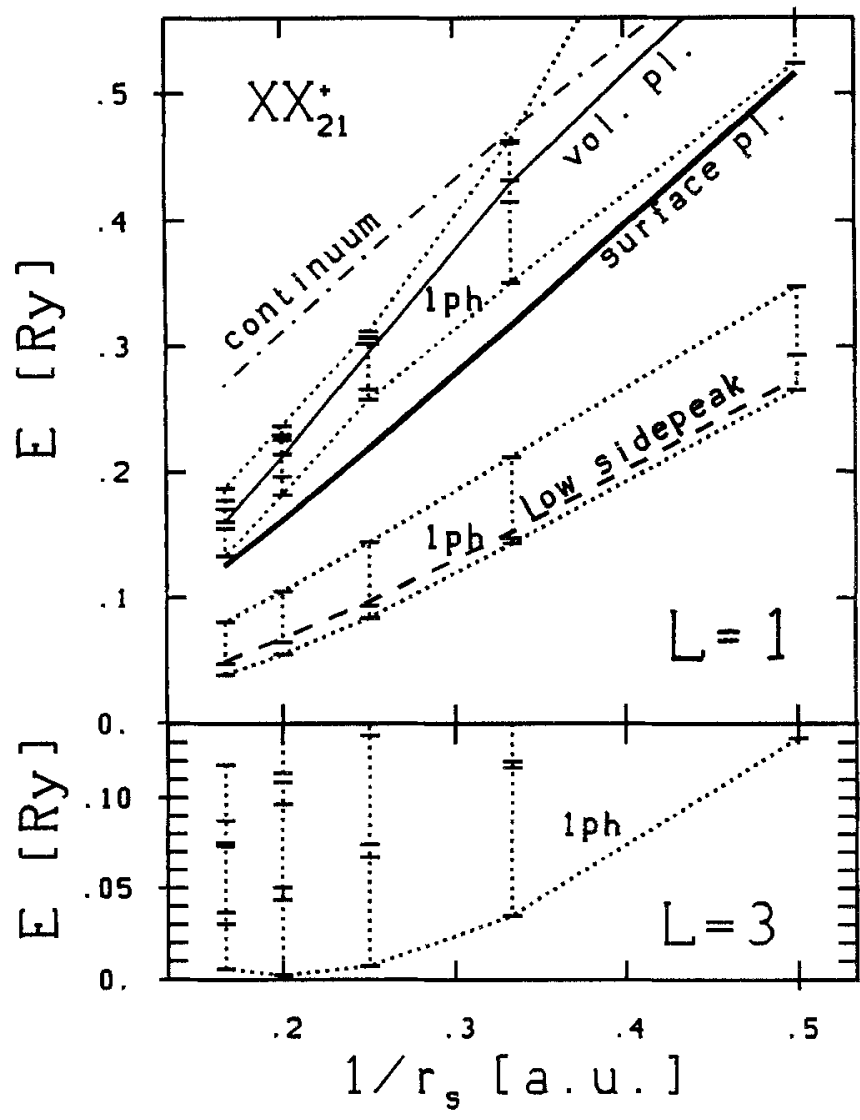

Fig. 2. Upper part: spectral properties for $L=1$ modes positively charged metal clusters with $N=21$ versus $r_{s}$; explanations as in Fig. 1. Lower part: the lower end of the low $1 p h$ band for $L=3$ modes

It persists also for resonances with higher angular momenta and for higher charge states. However, there are details in the low-energy spectra which depend sensitively on the model parameters. An example is given in the lower part of Fig. 2 where we show the low energy modes with $L=3$. There is a sensitive dependence on $r_{s}$ with a most dramatic development near $r_{s}=5 a$.u. . The energy of the lowest $1 p h$ state becomes nearly degenerate with the ground state. One should thus expect an extremely soft octupole vibration or even a stable octupole deformed ground state. It is to be noted, however, that this behaviour is extremely sensitive to changes in the model, as e.g. surface thickness in the jellium background. The interference of those low-energy electronic states with the phonon vibrations of the cluster will also play a crucial role. This, in turn, hints that experimental information on the low-energy spectra is highly desirable because it challenges theoretical models.

The gross features and the approximate positions of the resonances are well predicted by the Skyrme force for nuclei and the Gunnarson-Lundquist functional together with the jellium approach for metal clusters. The problems come, as usual, with the details. In nuclear, physics we have presently no working Skyrme parametrization which delivers a precise description of the nuclear ground-state properties together with appropriate resonance energies and low-energy spectra. The problem is most probably the zero-range of the Skyrme functionals and one may resolve them with finite-range functionals similar as it has been done in liquid ${ }^{3} \mathrm{He}[8,6]$. The

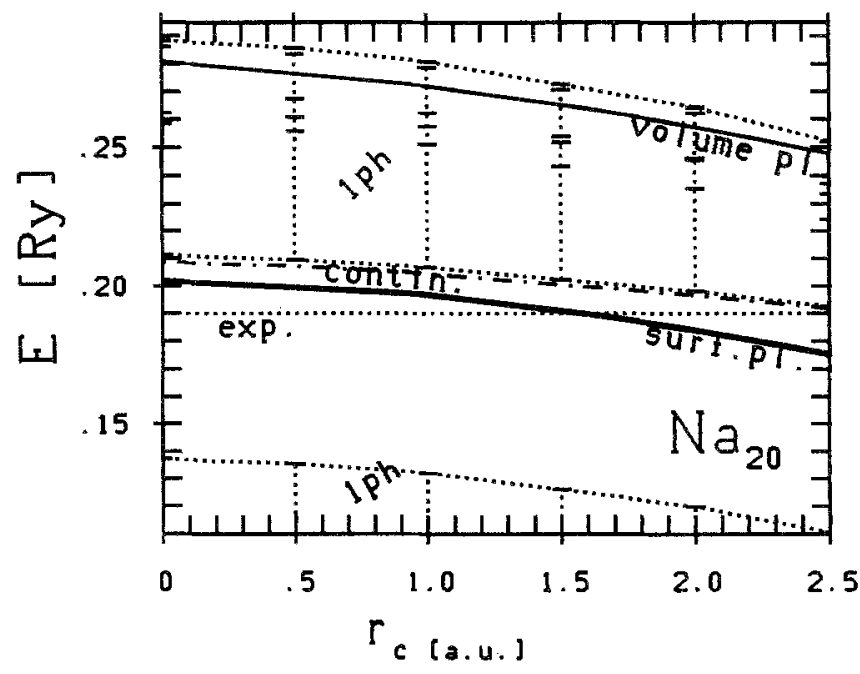

Fig. 3. Spectral properties for $L=1$ modes in neutral $\mathrm{Na}_{20}$ versus the pseudopotential core-radius $r_{c}$. Explanations as for Fig. 1. In addition comes the dotted line at $E \approx 0.19 R y$ to indicate the position of the experimental peak

energy-density functionals are on much better grounds in electron systems [9]. And yet, the position of the surface plasmon comes somewhat too high in calculations within the jellium approach with steep jellium surface. There is much debate about which approximation is to be blamed for that disease. There are strong arguments for a Self-InteractionCorrection (SIC) which aims to eliminate the double counting of one electron in the effective Hartree approximation of the Kohn-Sham scheme [10]. But that is not a unique explanation. There is, on the other hand, the observation that a finite surface width of the jellium can easily bring the resonance peak into the right position [11]. We show in Fig. 3 the spectral properties for $\mathrm{Na}_{20}$ as function of the surface width, given here from a folding of the jellium background with a pseudopotential of radius $r_{c}$. We see again a smooth dependence of all spectral features which confirm once more the notion of "jellium scaling". However, there is a clear trend of the energies which leads to a good agreement with the experimental peak energy at $r_{c} \approx 1.7 \mathrm{a}$.u. and that is just a typical value of the pseudopotential core-radius for $\mathrm{Na}$. This shows that the concept of a finite surface is a useful, and probably necessary, completion of the jellium approach. But it would be premature to sell this as the unique explanation for the proper positioning of the resonance energy. There are several more effects to be considered, as e.g. ionic structure or core polarization, which all will have their contribution to the resonance. The various strains on the resonance energy can be shown in the sum rule model of the surface plasmon. The resonance energy becomes in the sumrule model $\omega^{2}=\omega_{\text {surf }} \int \mathrm{d}^{3} r \rho_{e l} \rho_{I} / N \rho_{0}$ where $\rho_{e l}$ is the electron density and $\rho_{J}$ the ionic jellium density (both positive definite). The bulk value $\omega_{s r u f}$ for the surface plasmon is obtained in the limit of steep densities $\rho_{e l}=\rho_{I}=\rho_{0} \vartheta_{V o l}$ where $\vartheta_{V o l}$ is a step function on the systems volume. We expand the actual densities as $\rho=\rho_{0} \vartheta_{V o l}+\delta \rho$ for $\rho_{e l}$ and $\rho_{I}$ and obtain

$\omega^{2}=\omega_{s u r f}^{2}\left[1+\int \mathrm{d}^{3} r \vartheta_{V o l}\left(\delta \rho_{e l}+\delta \rho_{I}\right)+\int \mathrm{d}^{3} r \frac{\delta \rho_{e l} \delta \rho_{I}}{\rho_{0}}\right]$ 
The leading term is indeed the energy of the surface plasmon. The first order correction is determined by the portion of density which is missing inside the volume. It will be negative as both $\delta \rho$ are negative inside the volume (and positive outside for compensations). For $\delta \rho_{e l}$, this is the effect from the "electron spill-out" as discussed in [12]. There is obviously an additional effect from $\delta \rho_{I}$, exploring the ionic spill-out. But note that an ionic surface width counts twice as it increases also the electron spill-out. This explains why a soft jellium surface can lower the resonance energy so efficiently. The consideration also explains the success of the Self-Interaction-Correction: a softer Coulomb potential will equally well enhance the electron spill-out and thus lower the resonance energy. But both (competing) explanations are at stake if we consider ionic structure. For then the $\delta \rho_{I}$ is more than a small correction at the surface and provides wild oscillations throughout the whole volume. The problem is still awaiting a thorough investigation.

Finally, we want to remark on the splitting of the resonances due to deformation of the cluster or nucleus. The resonance is split in a deformed nucleus according to elongation or sqeezing of the principle axes changing the freqency $\propto 1 / R_{\text {axis }}$ which can be derived from scaling arguments employing a zero-range residual interaction. A similar splitting occurs for metal clusters, see e.g. [13]. But the size of the splitting is a factor of two smaller than in case of nuclei yielding a trend in frequency more like $\propto 1 / \sqrt{R_{a x i s}}$. This is due to the long range Coulomb force which weakens the scaling effects. We hope that this last example has shown again the interesting mix of similarities and differences in the dynamics of nuclei compared to metal clusters. One thing remains yet similar: both cases deserve further theoretical fine-tuning in the mean-field models to reach a reliable quantitative agreement with the data.

\section{References}

1. Gunnarson, O., Lundquist, B.I.: Phys.Rev. B13, 4274 (1976)

2. Bartel, J., Quentin, P., Brack, M., Guet, C., Hakanssson, H.B.: Nucl.Phys. A386, 79 (1982)

3. Reinhard, P.-G., Brack, M., Genzken, O.L: Phys.Rev. A41, 5568 (1990)

4. Reinhard, P.-G., Gambhir, Y.K.: Ann.Phys. (Leipzig) 1, 598 (1992)

5. Reinhard, P.-G.: Ann.Phys. (Leipzig) 1, 632 (1992)

6. Weisgerber, S., Reinhard, P.-G.: Ann,Phys. (Leipzig) (submitted)

7. Reinhard, P.-G., Genzken, O., Brack, M.: (in preparation)

8. Weisgerber, S., Reinhard, P.-G.: Phys.Lett. A158, 407 (1991)

9. Reinhard, P.-G.: Phys.Lett. A169, 281 (1992)

10. see the contributions of W. Ekardt and J.M. Pacheco in these proceedings

11. Rubio, A., Balbas, L.C., Alonso, J.A.: Z.Phys. D19, 93 (1991)

12. Brack, M.: Rev.Mod.Phys. (submitted)

13. Lauritsch, G., Reinhard, P.-G., Meyer, J., Brack, M.: Phys.Lett. A160, 179 (1991)

This article was processed by the author using the $\mathrm{LT}_{\mathrm{E}} \mathrm{X}$ style file pljour2 from Springer-Verlag. 